\title{
SEGMENTATION D'IMAGES AÉRIENNES PAR COOPÉRATION LPE-RÉGIONS ET LPE-CONTOURS. APPLICATION À LA CARACTÉRISATION DE TOITURES
}

\author{
Youssef El merabet ${ }^{1,2}$, Cyril Meurie $^{3}$, Yassine Ruichek ${ }^{1}$, Raja Touahni ${ }^{2}$, Abderrahmane Sbihi $^{4}$ \\ 1: IRTES-SET, UTBM, 90010 Belfort cedex - France \\ 2: Laboratoire LASTID, Faculté des Sciences, Université Ibn Tofail, BP 133, 14000 Kénitra - Maroc \\ 3: Univ Lille Nord de France, F-59000 Lille, IFSTTAR, LEOST, 59650 Villeneuve d'Ascq - France \\ 4: Laboratoire LABTIC, ENSA, Université Abdelmalek Essadi, Route Ziaten, km 10, BP 1818 Tanger - Maroc
}

\begin{abstract}
Résumé
Dans cet article, nous présentons une stratégie de segmentation d'images aériennes pour la caractérisation de toitures. Pour ce faire, une méthode de segmentation d'orthophotoplans basée sur une technique de coopération intégrant une segmentation par régions et une segmentation par contours est proposée. Les deux techniques de segmentation sont assurées par l'algorithme de la Ligne de Partage des Eaux (LPE). L'approche proposée est composée de trois étapes : (i) Une étape de pré-traitement qui consiste à prétraiter l'image initiale avec un couple invariant/gradient approprié et optimisé pour l'application. Pour cela, une série de tests permettant de choisir, d'une part, l'invariant colorimétrique le plus approprié parmi 24 invariants et, d'autre part, le meilleur gradient parmi 14 gradients issus de la littérature est réalisée. (ii) La deuxième étape comporte deux stratégies de segmentation par LPE. L'image pré-traitée est segmentée d'une part par une LPE-régions couplée à une stratégie de fusion de régions, et d'autre part, par une LPE-contours. Le processus de fusion de régions intègre des critères de fusion fondés sur des grandeurs radiométriques et géométriques adaptés aux particularités des orthophotoplans traités. Une technique de caractérisation 2D des arêtes de toitures par une analyse de segments est proposée afin de calculer l'un des critères de fusion. (iii) La troisième étape consiste à combiner les avantages de chaque méthode dans un même schéma de segmentation coopératif afin d'aboutir à un résultat de segmentation fiable. Les tests ont été effectués sur un orthophotoplan contenant 100 toitures de complexité variée et évalués avec le critère de VINET utilisant une segmentation de référence afin de prouver la robustesse et la fiabilité de l'approche proposée. La stratégie de coopération proposée permet d'aboutir à un taux de segmentation de $96 \%$ contre $83 \%$ pour une LPE-régions seule ou $88 \%$ pour une LPE-contours seule.
\end{abstract}

Mots clés : Invariants colorimétriques, Coopération régions-contours, Orthophotoplans, LPE, Fusion de régions.

\begin{abstract}
In this paper, an image segmentation strategy for roof modeling from aerial images is proposed. For that, an orthophotoplan segmentation method based on a cooperation technique including edge based segmentation and region based segmentation is proposed. Both segmentation techniques are assured by watershed algorithm. The proposed strategy is composed of three steps : (i) A preprocessing step that consists in preprocessing the input image with an appropriate couple of invariant/gradient optimized for the application. To this end, a series of tests allowing to choose the appropriate colorimetric invariant among a set of 24 invariants and define the best suitable gradient among a set of 14 gradients of the literature is carried. (ii) The second step is composed of two main treatments: On the one hand, the preprocessed image is segmented by the watershed regions coupled with an efficient regions merging technique. On the other hand, the preprocessed image is segmented by the watershed lines. The region merging process includes a merging criteria based on radiometric and geometrical features adapted to the orthophotoplan particularities. In order to calculate one of the merging criteria, a $2 D$ modeling of roof ridges is proposed. (iii) The third step consists in exploiting the advantages of each method and thus achieve reliable segmentation results. Experiments have been carried out on an orthophotoplan containing 100 roofs with varying complexity and evaluated with VINET criteria using a ground truth image in order to prove the robustness and the reliability of the proposed strategy. The proposed cooperation method produces a segmentation rate of $96 \%$ compared to $83 \%$ and $88 \%$ obtained with watershed regions and watershed lines, respectively.
\end{abstract}

Keywords : Colorimetric invariants, Regions-edges cooperation, Orthophotoplans, Watershed, Region merging.

\section{Introduction}

En traitement et analyse d'images, la littérature s'accorde à dire que segmenter une image revient à parti- tionner celle-ci en régions homogènes, c'est-à-dire en un ensemble de points connexes de l'image ayant des propriétés communes. Cette étape est réalisée en amont de traitements de plus haut niveau tels que la détec- 
tion d'objets ou la reconnaissance de formes. De nombreuses méthodes de segmentation ont vu le jour dans la littérature mais si l'on considère la richesse de la morphologie mathématique, nous distinguons deux grandes catégories que sont les approches contours et les approches régions. Les méthodes basées sur les contours cherchent à détecter les dissimilarités dans l'image. Elles supposent généralement un modèle à priori des discontinuités recherchées et opèrent de manière très localisée. Cette catégorie présente l'inconvénient d'être sensible au bruit, ce qui conduit généralement à l'obtention de contours non fermés (Kermad et Chehdi, 1998), (Liu et Yu, 2012). La deuxième catégorie relative aux méthodes basées régions cherchent à regrouper les pixels connexes en régions distinctes selon un critère de similarité donné. L'avantage de cette catégorie d'approches est qu'elle fournit une carte de régions (donc des zones fermées). Toutefois, la localisation des frontières des régions reste généralement peu précise (Sebari et $\mathrm{He}, 2007$ ), (Liu et $\mathrm{Yu}, 2012$ ). Dans la plupart des cas, ceci peut être justifié par la difficulté de définir des critères et/ou des paramètres adaptés pour effectuer la fusion/division des régions ou l'agrégation des pixels (Bellet et al., 1995), (Sebari et He, 2009). En effet, ces critères et/ou paramètres sont généralement calculés par un mécanisme essais-erreurs.

D'une manière générale, les méthodes de segmentation issues de ces deux catégories ont de nombreux avantages mais connaissent aussi des limites en termes de performance, plus particulièrement lorsqu'elles sont utilisées pour des applications spécifiques comme cela est le cas ici pour la caractérisation de toitures à partir d'images aériennes. L'intégration de plusieurs techniques dans une même stratégie semble donc être la meilleure voie à suivre. Ceci permettrait d'exploiter les avantages de chacune de ces méthodes, de pallier leurs inconvénients lorsqu'elles sont utilisées indépendamment, et par conséquent d'améliorer la qualité de la segmentation finale. Dans ce contexte, plusieurs approches de segmentation d'images combinant les résultats issus de techniques différentes ont vu le jour ces dernières années (Sebari et He, 2007), (Jayadevappa et al., 2009). Malheureusement, le choix de la méthode de segmentation à utiliser ou à combiner est fortement dépendant des particularités des images à traiter ainsi que de l'application considérée. La majorité des approches de segmentation ne sont pas adaptées pour des images aériennes et satellitaires (de très haute résolution spatiale, spectrale et temporelle). C'est pourquoi une grande variété de méthodes de segmentation d'images aériennes ont été développées et souvent combinées ces dernières années. La plupart de ces techniques consistent à détecter des objets d'intérêt tels que les routes (Anil et Natarajan, 2010; Volodymyr et Geoffrey, 2010), ou les bâtiments (Hazelhoff et With, 2011; Angiati et Dellepiane, 2011), etc. Bien que ces études aient été publiées dans le cadre de la segmentation d'images aériennes ou satellitaires, et plus spécifiquement pour la détection/extraction de bâtiments, aucune d'entre elles n'abordent le problème de la segmentation fine des toits des bâtiments (différentes parties constituant la toiture) en vue de la caractérisation des toitures qui joue un rôle crucial dans le domaine de la génération automatique de bâtiments 3D.

Les travaux présentés dans cet article s'inscrivent donc dans une approche globale qui consiste à extraire à partir d'images aériennes, des informations relatives aux toitures de bâtiments. Ceci permettra, in fine, de pouvoir reconnaître une toiture par rapport à une base de connaissances et ainsi affiner/déformer des modèles 3D générés automatiquement à partir de données géographiques. Dans cet article, nous nous focalisons sur la mise en place d'une stratégie permettant d'obtenir une segmentation de la toiture et des éléments la constituant la plus précise possible. Pour ce faire, nous proposons une stratégie de segmentation basée sur la coopération de Lignes de Partage des Eaux régions et contours (LPErégions/LPE-contours).

Cet article est organisé comme suit. La section 2 décrit la méthodologie de l'approche de segmentation d'orthophotoplan proposée. Dans la section 3, nous proposons une étape de prétraitement de l'image par sélection d'un couple invariant colorimétrique/gradient. Dans la section 4, deux stratégies de segmentation (par croissance de régions et par contours) sont décrites. Dans cette section, nous détaillons : 1 / la segmentation initiale de l'image prétraitée, basée sur l'utilisation d'une LPErégions, $2 /$ une stratégie de caractérisation des arêtes de toiture, 3 / le processus de fusion de régions permettant d'améliorer la qualité de la segmentation initiale, 4/ la segmentation de l'image prétraitée basée sur l'utilisation d'une LPE-contours. Dans la section 5, nous décrivons la stratégie de coopération entre les deux segmentations par LPE-régions et LPE-contours. Avant de conclure, nous présentons dans la section 6 , les résultats expérimentaux obtenus sur un orthophotoplan contenant 100 toitures de complexité variée.

\section{Méthodologie}

L'approche de segmentation d'orthophotoplans proposée dans cet article se positionne dans la catégorie des méthodes de segmentation dites hybrides. En effet, la complémentarité des informations obtenues à partir des contours et des régions contribue à une meilleure prise en compte des caractéristiques des entités de l'image et, par conséquent, à une meilleure segmentation des objets. Plusieurs approches coopératives ont été proposées dans la littérature et trois catégories peuvent être distinguées (Sebari et $\mathrm{He}, 2007$ ) : 1 / la coopération séquentielle; 2 / la coopération mutuelle; $3 /$ la coopération des résultats. Comme cela est rappelé par SEBARI, ces différentes approches se distinguent généralement par la façon et le niveau de traitement dans lequel l'intégration des méthodes est réalisée. Soit deux méthodes de segmentation différentes. La première catégorie de méthodes coopératives regroupe les approches pour lesquelles une première segmentation est réalisée par la première méthode, et le résultat de celle-ci est en- 
suite utilisé dans la deuxième technique de segmentation (Muñoz et al., 2000; Mueller et al., 2004). La deuxième catégorie revient à exécuter les deux méthodes de segmentation en parallèle tout en permettant les échanges d'informations entre elles (Salotti, 1994; Bellet et al., 1995). La dernière catégorie consiste à exécuter les deux méthodes de segmentation de manière totalement indépendante et à fusionner les résultats obtenus par chacune d'entre elles (Chu et Aggarwal, 1993; Zugaj et Lattuati, 1998). L'approche coopérative de segmentation d'orthophotoplans proposée dans cet article appartient aux approches hybrides par fusion de résultats. Elle consiste à intégrer deux stratégies de segmentation basées sur l'algorithme de la Ligne de Partage des Eaux, mais considérant pour l'une, une approche régions (LPE-régions) et pour l'autre, une approche contours (LPE-contours). La figure 1 illustre le synopsis général de l'approche coopérative de segmentation d'orthophotoplans proposée. La méthodologie est composée de trois étapes principales introduisant plusieurs traitements présentés et détaillés ci-après.

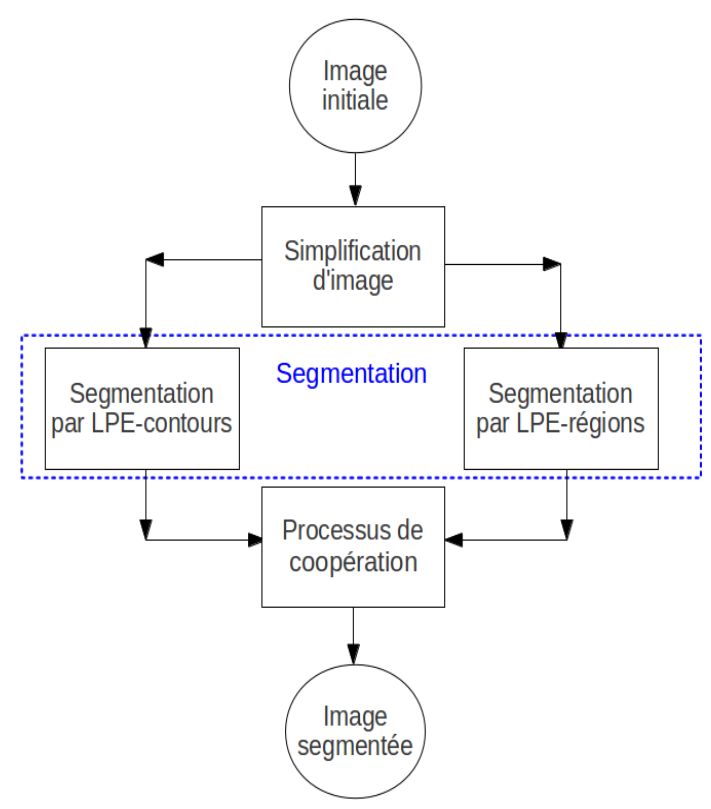

FIGURE 1: Synopsis général de l'approche coopérative de segmentation d'orthophotoplans proposée.

1. Prétraitement de l'image : cette partie consiste à prétraiter l'image originale avec un couple invariant/gradient adapté et optimisé pour l'application. L'utilisation de ce couple invariant/gradient permet de limiter les artefacts affectant les orthophotoplans et à les rendre invariants aux changements de luminosité et aux ombres, afin de permettre une segmentation précise des différents éléments constituant une toiture (pans, cheminées, fenêtres, chiens assis, etc.).

2. Segmentation par LPE : cette partie est composée de deux stratégies de segmentation d'image par LPE, totalement indépendantes et s'exécutant en parallèle. Dans une première phase, l'image pré- traitée est partitionnée en régions par application d'une LPE-régions couplée à une stratégie efficace de fusion de régions. Le processus de fusion de régions intègre des critères de fusion fondés sur des grandeurs radiométriques et géométriques en relation avec les particularités des orthophotoplans traités. Les grandeurs radiométriques sont la moyenne intérieure des régions et le contraste sur les frontières communes entre deux régions candidates à la fusion. Les grandeurs géométriques sont définies en considérant la nature rectiligne des arêtes de toits des bâtiments. La modélisation des toits par des arêtes est obtenue par une caractérisation 2D, présentée en section 4. Dans une deuxième phase, exécutée en parallèle, l'image prétraitée est segmentée par une LPE-contours sans aucune étape de post-traitement.

3. Stratégie de coopération : cette dernière étape consiste à intégrer les résultats issus des deux stratégies de segmentation par LPE (régions et contours de l'étape précédente) dans un même schéma de segmentation. Le processus de coopération exploite le bénéfice de chaque méthode de segmentation pour aboutir à un résultat de segmentation plus robuste en termes de détection des éléments constituant une toiture, à savoir les pans, cheminées, fenêtres, etc.

\section{Prétraitement de l'image}

Les images (orthophotoplans) dont nous disposons présentent une certaine hétérogénéité en termes d'illuminations, ombres, etc., qui rendent la segmentation de l'image et l'extraction des zones d'intérêt complexe. Afin de pallier ces inconvénients, nous rejoignons la stratégie de nombreux auteurs en prétraitant l'image par l'application d'un invariant colorimétrique adapté (Gevers et Smeulders, 1997, 1999; Cong et al., 2010; El-merabet et al., 2011a). En effet, ces dernières années, l'invariance couleur a suscité beaucoup d'intérêt et continue à intéresser le domaine de la vision par ordinateur. A titre d'exemple, nous pouvons citer l'utilisation d'invariant colorimétrique pour améliorer le suivi de points d'intérêt dans les images couleur (Gouiffès, 2005), la mise en correspondance d'images (Gevers et Smeulders, 1999), l'estimation de mouvement dans des séquences vidéo (Golland et Bruckstein, 1997), l'extraction d'attributs et la ré-identification de personnes dans un environnement de transport (Cong et al., 2010), la détection de bâtiments dans les images aériennes (Sirmacek et Unsalan, 2008), etc. Nous pensons alors que, dans le cadre de notre application, l'extraction des différents objets constituant une toiture pourrait être améliorée par l'utilisation d'un invariant colorimétrique adapté.

La première étape de l'approche de segmentation d'orthophotoplans proposée dans cet article est donc le prétraitement de l'image initiale par un couple invariant/gradient approprié. L'utilisation d'un invariant colorimétrique permettrait probablement de limiter l'effet 
des changements brusques de luminosité ou ombres présentes sur les images aériennes. Mais nous parlerons ici d'un couple invariant/gradient car tout comme l'utilisation d'une image prétraitée (par un invariant), l'image de gradient constitue une entrée corrélée pour l'algorithme de segmentation par LPE. II convient donc de choisir un couple invariant/gradient pour optimiser cette première étape de segmentation. La figure 2 illustre le synopsis de la stratégie proposée permettant de choisir le couple optimal invariant/gradient, à utiliser en entrée de la LPE. Cette stratégie se décompose en plusieurs étapes. La première étape consiste à appliquer l'invariant colorimétrique candidat sur l'image initiale. La deuxième étape se divise en deux sous-étapes selon que l'on choisisse de s'intéresser à l'information disponible en niveaux de gris (composante couleur d'un invariant colorimétrique/gradient niveaux de gris) ou en couleur (invariant colorimétrique/gradient couleur). Dans le premier cas, nous extrayons les trois composantes couleur de l'image prétraitée par l'invariant colorimétrique, puis nous calculons le gradient image sur chaque composante ainsi extraite. Dans le deuxième cas, nous appliquons directement le gradient couleur sur l'image prétraitée par l'invariant colorimétrique. L'étape suivante consiste à effectuer une sélection des minima locaux afin de disposer en sortie de ces traitements, de germes et du gradient à utiliser en entrée de l'algorithme de segmentation par LPE. A l'issue de ce dernier, la qualité de l'image ainsi segmentée est ensuite évaluée avec le critère de VINET, nécessitant une segmentation de référence.

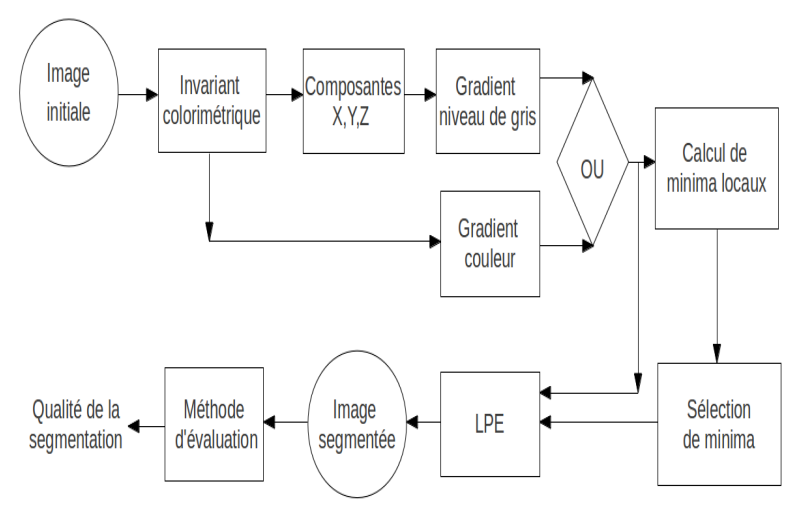

FIGURE 2: Synopsis de la stratégie du choix du couple optimal invariant/gradient.

Notre étude sur le choix d'un invariant colorimétrique adapté à notre application s'est faite sur une base de 24 invariants de la littérature regroupés dans le tableau 1 et classés comme suit : i) espaces tridimensionnels composés de trois composantes couleur $(X, Y, Z)$, ii) espaces bidimensionnels composés de deux composantes couleur $(X, Y)$, ou iii) unidimensionnel composés que d'une seule composante couleur $X$.

Nous montrerons dans la section 6 l'apport de l'utilisation de chacun de ces invariants colorimétriques dans la phase de pré-traitement des images de toitures de notre application. Mais avant toute chose, il nous a semblé important de rappeler ici la définition des quatre meilleurs invariants illustrés sur la figure 3.

La normalisation de Greyworld : normalise chaque pixel par la moyenne de l'image (Buchsbaum, 1980).

$$
\begin{aligned}
\left(R^{*}=\frac{R}{\operatorname{mean}(R)}, G^{*}\right. & \left.=\frac{G}{\operatorname{mean}(G)}, B^{*}=\frac{B}{\operatorname{mean}(B)}\right) \\
\operatorname{mean}(R) & =\frac{1}{N * M} \sum_{i=0}^{N-1} \sum_{j=0}^{M-1} r_{i j}, \\
\operatorname{mean}(G) & =\frac{1}{N * M} \sum_{i=0}^{N-1} \sum_{j=0}^{M-1} g_{i j} \\
\operatorname{mean}(B) & =\frac{1}{N * M} \sum_{i=0}^{N-1} \sum_{j=0}^{M-1} b_{i j}
\end{aligned}
$$

où $r_{i j}, g_{i j}$ et $b_{i j}$ sont respectivement les valeurs des pixels aux rang(i,j) de la composante rouge,verte et bleue. $\mathrm{M}$ et $\mathrm{N}$ correspondent à la largeur et la hauteur de l'image.

La normalisation Affine : normalise la couleur de l'image par l'équation suivante (Fusiello et al., 1999) :

$$
f^{K}(p)=\frac{I^{K}(p)-\operatorname{mean}\left(I^{K}(p)\right)}{s t d\left(I^{K}(p)\right)}, K=R, G, B,
$$

où $\operatorname{std}\left(I^{K}\right)$ est l'écart-type de $f^{K}(p)$ calculé dans une fenêtre d'intérêt $\mathcal{W}$ centrée sur le pixel $p$.

La normalisation MaxRGB : par analogie avec la normalisation de Greyworld, la normalisation MaxRGB normalise l'image par un pixel de référence (Finlayson et al., 2005).

$$
\left(R^{*}=\frac{R}{\operatorname{Max}(R)}, G^{*}=\frac{G}{\operatorname{Max}(G)}, B^{*}=\frac{B}{\operatorname{Max}(B)}\right)
$$

La normalisation Maximum-intensité : normalise également la couleur de l'image selon la relation suivante (Dargham, 2008) :

$$
\begin{aligned}
& \left(R^{*}=\frac{R}{M a x_{r g b}}, G^{*}=\frac{G}{M a x_{r g b}}, B^{*}=\frac{B}{M a x_{r g b}}\right) \\
& \text { avec } \operatorname{Max}_{r g b}=\operatorname{Max}(\mathrm{R}+\mathrm{G}+\mathrm{B})
\end{aligned}
$$

L'algorithme de LPE nécessite une image de gradient que nous proposons de calculer à partir de l'image prétraitée. Nous présentons dès à présent les différents gradients que nous avons considérés dans la section 6 pour le choix du couple optimal invariant/gradient.

Afin d'étudier l'influence de l'image de gradient dans la segmentation par LPE-régions et par conséquent, définir le meilleur gradient pour notre approche de segmentation des orthophotoplans, plusieurs méthodes de calcul de gradient issues de la littérature ont été utilisées et testées. Ces méthodes peuvent être classées en deux 


\begin{tabular}{|c|c|c|c|}
\hline \multicolumn{2}{|c|}{ Invariants Colorimétriques } & Abbréviations dans les figures 11,12 & Références \\
\hline Espaces tridimensionnels & $\begin{array}{l}\text { - la normalisation de Greyworld } \\
\text { - RGB-rang } \\
\text { - les attributs Color Constant Color Indexing } \\
\text { - MaxRGB } \\
\text { - la normalisation d'intensité } \\
\text { - la normalisation Affine } \\
\text { - la normalisation compréhensive } \\
\text { - les attributs c1c2c3 } \\
\text { - m1m2m3 } \\
\text { - I1I2|3 } \\
\text { - les attributs }|4| 5 \mid 6 \\
\text { - c4c5c6 } \\
\text { - les attributs CrCgCb } \\
\text { - m4m5m6 } \\
\text { - A1A2A3 } \\
\text { - la norme L2 } \\
\text { - la normalisation Maximum-Intensité } \\
\text { - l'espace hsl }\end{array}$ & $\begin{array}{l}\text { - Greyworld } \\
\text { - CCCI } \\
\text { - Chromaticity } \\
\text { - Affine } \\
\text { - Comprehensive }\end{array}$ & $\begin{array}{l}\text { - (Schaefer, 2004) } \\
\text { - (Finlayson et al., 2005) } \\
\text { - (Finlayson et al., 2005) } \\
\text { - (Finlayson et al., 2005) } \\
\text { - (Finlayson et al., 2005) } \\
\text { - (Fusiello et al., 1999) } \\
\text { - (Schaefer, 2004) } \\
\text { - (Gevers et Smeulders, 1999) } \\
\text { - (Gevers et Smeulders, 1999) } \\
\text { - (Gevers et Smeulders, 1999) } \\
\text { - (Latecki et al., 2005) } \\
\text { - (Latecki et al., 2005) } \\
\text { - (Gouiffès, 2005) } \\
\text { - (Gouiffès, 2005) } \\
\text { - (Gouiffès, 2005) } \\
\text { - (Gouiffès, 2005) } \\
\text { - (Dargham, 2008) }\end{array}$ \\
\hline Espaces bidimensionnels & $\begin{array}{l}\text { - les coordonnées réduites } \\
\text { - l'espace } \mathrm{CrCb} \\
\text { - les attributs de couleurs opposés } 0102\end{array}$ & & $\begin{array}{l}\text { - (Gevers et Stockman, 2004) } \\
\text { - (Gevers et Smeulders, 1997) } \\
\text { - (Gevers et Smeulders, 1997) }\end{array}$ \\
\hline Espaces unidimensionnels & $\begin{array}{l}\text { - la saturation S } \\
\text { - la teinte H } \\
\text { - Log-Hue }\end{array}$ & & $\begin{array}{l}\text { - (Gevers et Smeulders, 1999) } \\
\text { - (Gevers et Smeulders, 1999) } \\
\text { - (Finlayson et al., 2005) }\end{array}$ \\
\hline \multicolumn{4}{|c|}{ Opérateurs de Gradient } \\
\hline Gradients niveaux de gris & $\begin{array}{l}\text { - la dérivée première de l'image } \\
\text { - gradient morphologique niveaux de gris } \\
\text { - NonMaximaSuppression } \\
\text { - Sobel } \\
\text { - Roberts } \\
\text { - Prewitt } \\
\text { - Deriche } \\
\text { - Shen }\end{array}$ & $\begin{array}{l}\text { - GradientF } \\
\text { - GradientM }\end{array}$ & \\
\hline Gradients couleur & $\begin{array}{l}\text { - le gradient marginal } \\
\text { - le gradient morphologique couleur } \\
\text { - le gradient Sobel couleur } \\
\text { - le gradient Sobel TLS } \\
\text { - l'opérateur de Carron } \\
\text { - le gradient de Di-Zenzo }\end{array}$ & $\begin{array}{l}\text { - GradientC } \\
\text { - GradientMC } \\
\text { - SobelC } \\
\text { - SobelTLS }\end{array}$ & $\begin{array}{l}\text { - (Lezoray et al., 2007) } \\
\text { - (Carron, 1995) } \\
\text { - (Carron, 1995) } \\
\text { - (Carron, 1995) } \\
\text { - (Di-Zenzo, 1986) }\end{array}$ \\
\hline
\end{tabular}

TABLE 1: Tableau illustrant les 24 invariants testés. 


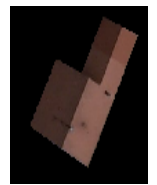

(a)

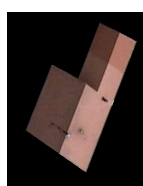

(b)

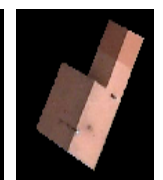

(c)

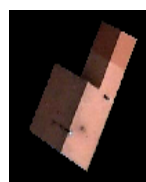

(d)

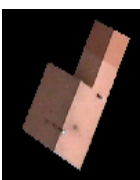

(e)
FIGURE 3: Exemple d'une toiture sous différents invariants colorimétriques ((a) sans invariant colorimétrique, (b), (c), (d) et (e) avec les normalisations de Greyworld, MaxRGB, Affine et Maximum-intensité).

catégories, à savoir les méthodes calculant le gradient niveaux de gris et les méthodes calculant le gradient couleur. Pour la catégorie des gradients niveaux de gris, les opérateurs peuvent être simples comme : 1/ la dérivée première de l'image; 2 / le gradient morphologique niveaux de gris obtenu par différence entre l'image dilatée et l'image érodée; 3/ NonMaximaSuppression (le principe étant de ne conserver de l'image d'amplitude du gradient que les valeurs qui sont maximales dans la direction orthogonale au gradient) ; 4/ Sobel ; 5/ Roberts et 6/ Prewitt ; ou plus complexes comme : 7/ Deriche et 8/ Shen. Quant à la deuxième catégorie relative aux gradients couleur, nous avons choisi de tester des gradients issus des trois approches principales : les méthodes scalaires; les méthodes perceptuelles et les méthodes vectorielles. Nous avons alors utilisé : $1 /$ le gradient marginal, 2/ le gradient morphologique couleur obtenu par différence entre l'image dilatée et l'image érodée en utilisant l'ordre lexicographique, 3/ le gradient Sobel couleur calculé sur l'image RGB, 4/ le gradient Sobel couleur calculé dans l'espace couleur TLS, 5/ l'opérateur de CARRON et 6 / le gradient de DI-ZENZO.

\section{Segmentation par LPE}

Face aux différentes méthodes de segmentation d'images existantes, nous avons choisi de nous placer dans le cadre de la morphologie mathématique. L'une des techniques les plus puissantes de cette catégorie est la Ligne de Partage des Eaux (LPE). En effet, l'algorithme de la LPE offre l'avantage d'obtenir des régions fermées dont les frontières coïncident dans la plupart des cas avec les contours les plus significatifs de l'image. Bien que la LPE soit habituellement considérée comme une approche de segmentation par croissance de régions, SMET et al. (Smet et al., 2000) ont souligné que la transformation par LPE s'avère être un outil de segmentation puissant qui peut être attribué à la fois aux techniques de segmentation par contours et aux techniques de segmentation par croissance de régions. De nombreux algorithmes séquentiels ont été développés dans la littérature et peuvent être classés en deux catégories. L'une porte sur la spécification d'algorithmes récursifs (Vincent et Soille, 1991); l'autre est basée sur des fonctions de distance (topographique) (Meyer, 1991). Pour plus de détails à ce sujet, ROERDINK et MEIJSTER présentent une étude approfondie sur les algorithmes de LPE et leur implémentation (Roerdink et Mei- jster, 2001). Dans le cadre de la détection de toitures à partir d'images aériennes, nous proposons une stratégie de coopération entre une segmentation par LPE-régions et une segmentation par LPE-contours afin d'obtenir une meilleure segmentation des toitures et des éléments qui les composent. Nous détaillerons dans la section suivante notre stratégie de segmentation par LPE-régions (algorithme de MEYER), couplée à une technique de fusion de régions utilisant la caractérisation des arêtes de toitures. Nous présenterons ensuite la segmentation par LPE-contours (algorithme de VINCENT \& SOILLE).

\subsection{Segmentation initiale par LPE-régions}

Comme indiqué précédemment, une fois le prétraitement de l'image réalisé, nous cherchons à segmenter l'image par une LPE-régions. Cette technique bien connue de la morphologie mathématique repose sur une heuristique simple qui consiste à analyser les pixels par ordre croissant de niveaux de gris. Cet algorithme fait partie des algorithmes des LPE inter-pixel (par inondation) dont nous rappelons le principe de fonctionnement ci-après (Meyer, 1991) :

Etant donnée une image I, le principe consiste à étendre le plus possible les minima locaux de l'image en utilisant l'ordre de priorité donné par une image de potentiel ou de gradient. Les pixels les moins élevés sont traités en priorité. Les quatre étapes principales de cet algorithme sont décrites ci-après :

1. Attribuer une étiquette à chaque minimum de l'image ; marquer chaque point appartenant à un minimum avec l'étiquette correspondant à ce minimum. Initialiser un ensemble $S$ de type file d'attente hiérarchique à l'ensemble vide.

2. Insérer chaque point étiqueté dans l'ensemble $S$. A chaque insertion, la file établit un tri des points selon la priorité correspondant à leur altitude (le module du gradient correspondant).

3. Extraire de l'ensemble S le point $p_{1}$ le plus prioritaire d'altitude minimale (de faible gradient), c'està-dire $I\left(p_{1}\right)=\min \{I(p) \mid p \in S\}$. Attribuer à chaque point $p$ adjacent à $p_{1}$ (dans un voisinage $\mathcal{V}=8$-connexité) et non-étiqueté l'étiquette de $p_{1}$ et insérer $p$ dans $\mathrm{S}$. La priorité d'insertion du point $\mathrm{p}$ correspond à son altitude, à l'instar de l'initialisation. Le point $p_{1}$ est ensuite détruit de la file d'attente hiérarchique.

4. Répéter l'étape 3 tant que S n'est pas totalement vide.

Cette technique offre généralement de bons résultats, mais a tendance à produire une sur-segmentation à cause d'un nombre de germes trop important. Plusieurs solutions ont été proposées dans la littérature afin de pallier cet inconvénient. La plupart d'entre elles consistent à : 1 / prétraiter l'image initiale ; 2 / sélectionner un ensemble réduit de minima locaux; 3 / procéder à une fusion des régions non-significatives. Ces solutions ont toutes été prises en considération dans notre 
approche de segmentation des orthophotoplans. En effet, l'image initiale est prétraitée en appliquant un invariant colorimétrique adapté. En ce qui concerne la sélection de germes, plusieurs algorithmes ont été proposés dans la littérature pour réduire le nombre de minima locaux non-pertinents. La plus simple des méthodes est la sélection interactive des germes initiaux par l'utilisateur (Yan et al., 2006) ou en utilisant une connaissance à priori de l'image (Meyer et Beucher, 1990). L'approche dynamique introduite dans (Grimaud, 1992) consiste quant à elle à fournir un schéma de sélection intuitif contrôlé par un seul paramètre $\tau$ à l'aide de la reconstruction en niveau de gris. Dans le cadre de notre approche, nous avons opté pour un opérateur de sélection de minima locaux piloté par deux paramètres $\alpha$ et $\beta$ définissant le pourcentage de germes à conserver (60\% pour notre application) (Cohen et al., 2010).

La figure 4 décrit le principe de sélection de germes assuré par cet opérateur, que nous pouvons retrouver dans (Cohen et al., 2010), qui consiste à garder alpha germes de chaque beta-uplet. II est à noter que la diminution du nombre de minima locaux garantit une bonne réduction de la sur-segmentation et par conséquent, une meilleure segmentation de l'image. Toutefois, la sous-segmentation peut être produite lorsque les critères adoptés pour le filtrage des germes sont trop sélectifs. La figure 5 illustre quelques résultats de segmentation avec différents pourcentage de germes. Nous pouvons y voir clairement, l'effet de réduction de germes sur le résultat de segmentation. La segmentation générée à partir de tous les minima locaux, initialisant chacun une croissance de région (cf. figure $5 b$ ), s'avère très médiocre car l'image est fortement sur-segmentée en un grand nombre de régions homogènes (168 régions) qui ne sont pas toutes significatives. En revanche, l'image est sous-segmentée (plusieurs pans de toitures sont perdus) dans le cas où le nombre de minima locaux est trop réduit (cf. figure $5 \mathrm{e}$ ). Notons que le couple $(\alpha=10, \beta=15)$ semble être un bon compromis entre une atténuation suffisante de sur-segmentation et une restitution correcte des structures principales de la toiture (pas de sous-segmentation de l'image). En effet, la segmentation correspondante s'avère pertinente car la plupart des pans y sont présents avec une faible sur-segmentation de la toiture (ie 14 régions seulement). Ainsi, les résultats finaux de segmentation qui seront générés par l'approche de segmentation d'orthophotoplans proposée dans ce manuscrit, sont calculés à partir des minima locaux filtrés par ce couple.

Bien que la résolution du problème de sursegmentation soit améliorée avec cette stratégie de sélection de germes, elle peut encore être optimisée par l'ajout d'une étape de fusion de régions. Nous procédons alors à l'application d'une stratégie efficace de fusion de régions faisant intervenir des grandeurs radiométriques et géométriques basées sur les particularités des orthophotoplans traités. Une technique de caractérisation 2D des arêtes de toit est donc proposée en section 4.2

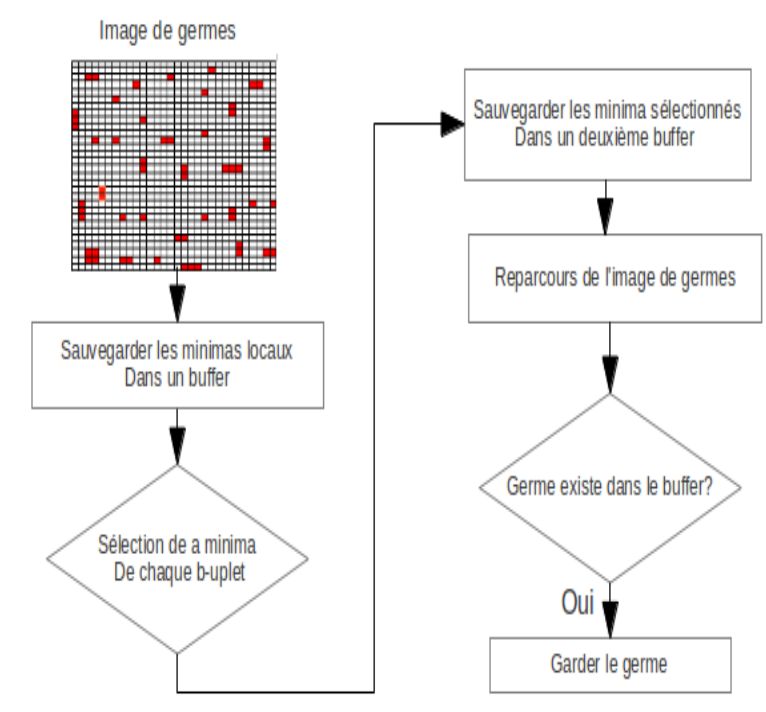

FiguRE 4: Illustration de l'opérateur de sélection de germes (Cohen et al., 2010).

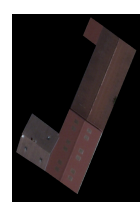

a

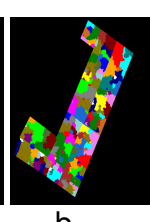

b

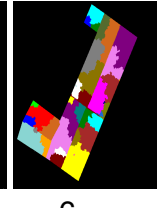

C

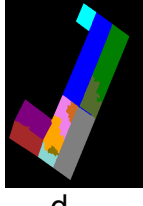

d

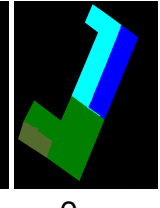

e
FIGURE 5: Exemple de réduction de germes ((a) l'image initiale; les images segmentées avec (b) tous les minima locaux (168 régions); (c) $\alpha=1, \beta=5$ (40 régions); (d) $\alpha=10, \beta=15$ (14 régions); (e) $\alpha=10, \beta=80$ (sous-segmentation avec 6 régions seulement)).

afin d'être utilisée dans le calcul du critère de fusion.

\subsection{Caractérisation des arêtes des toitures}

Considérant une image segmentée en régions, la caractérisation des arêtes des toitures consiste à définir un modèle $2 \mathrm{D}$ composé de plusieurs segments décrivant les arêtes de toits. Nous précisons que le terme segment introduit ici représente de points linéaires constituant un segment de droite. Le processus de caractérisation est basé sur le résultat de la segmentation préliminaire obtenue par la LPE-régions avec application du meilleur couple invariant/gradient. Dans un premier temps, cela consiste à extraire les frontières des régions de l'image pré-segmentée. Dans un deuxième temps, l'information sur les frontières extraites est exploitée pour transformer les arêtes de toits en segments. Notons que chaque arête de toits n'est caractérisée que par un seul et unique segment (les extrémités correspondent aux nœuds reliant l'arête). Les relations de jonction entre ces segments (arêtes) permettent de mettre en évidence les nœuds. Cette étape d'extraction de segments est suivie de plusieurs sous étapes permettant d'améliorer le modèle de segments : la coupure de segments, l'amélioration des intersections, le regroupement ou la jointure de points proches, etc. Les principales étapes de cette technique de caractérisation sont énoncées ci-après, mais 
le lecteur désireux d'avoir plus d'informations à ce sujet pourra consulter les travaux suivants : (El-merabet et al., 2011b, 2012).

1. L'extraction des frontières des régions de l'image segmentée préalablement par une LPE-régions s'effectue en balayant l'image suivant les directions $x$ et $y$ (horizontale et verticale). Tout pixel se trouvant dans un état de transition entre deux régions différentes est considéré comme pixel frontière (cf. figure 7c). A cela s'ajoute une étape d'amincissement pour réduire l'épaisseur des frontières à 1pixel.

2. L'extraction des segments et des nœuds est mis en œuvre grâce à un processus récursif qui consiste à choisir arbitrairement un pixel frontière comme pixel de départ, puis à construire un segment en considérant récursivement les pixels voisin, jusqu'à ce qu'il n'ait plus de pixels voisins non affectés. Le dernier pixel visité constitue alors le premier nœud $\mathrm{n} 1$ du segment. Le processus est réitéré en inversant le sens de parcours, en commençant cette fois-ci à partir du point noeud $\mathrm{n} 1$. A l'issue de la deuxième passe on obtient alors le second point nœud n2 du segment. Au cours du processus de recherche des points nœud, une procédure de sélection a été mise en place afin de savoir quelle direction privilégier lorsque de multiple pixels sont candidats. C'est le cas notamment lorsque le pixel courant relie plusieurs arêtes.

3. La subdivision de segments consiste à modifier/optimiser la position des nœuds reliant plusieurs arêtes afin de subdiviser les segments ne caractérisant pas la forme réelle d'une toiture. Pour chaque segment, nous cherchons l'ensemble des nœuds à l'intérieur de ce segment permettant de maximiser la somme des longueurs entre les différents segments (définis par ces nœuds deux à deux).

Une illustration de la caractérisation des arêtes de toitures par des segments est donnée sur la figure 6 . Sur cette figure, et toutes celles du manuscrit décrivant des régions, les couleurs de différentes régions de l'image sont choisies de manière aléatoire et ont pour seul but de permettre de mieux les identifier. En effet, pour plus de visibilité, les auteurs ont fait le choix de montrer le résultat du processus sous la forme d'une carte de régions (chaque pixel se voit attribuer un numéro correspondant au label de la région à laquelle il appartient).

\subsection{Fusion de régions}

La caractérisation 2D des arêtes de toitures étant maintenant effectuée, nous allons présenter l'algorithme de fusion de régions basé sur cette caractérisation et sur l'analyse du graphe d'adjacence de régions (RAG) de l'image préalablement segmentée (cf. section 4.1). L'analyse des résultats de segmentation obtenus par LPE-régions amène une remarque très importante. En

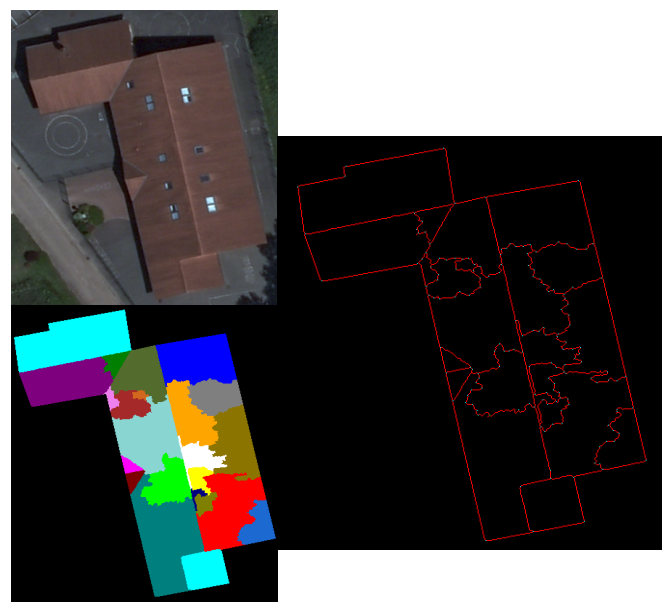

FIGURE 6: Exemple de caractérisation des arêtes des toitures (l'image initiale, l'image segmentée par LPE-régions et le modèle 2D correspondant).

effet, il est important de noter que suite à la segmentation faite par LPE, la nature rectiligne d'une frontière séparant deux régions de deux pans de toitures est toujours respectée, comme l'illustrent les régions labélisées 2 et 3 dans la figure $7 e$ où la frontière est modélisée au maximum par deux segments. Par contre, cela n'est pas le cas lorsqu'il s'agit d'une frontière séparant deux régions issues d'une sur-segmentation, comme l'illustrent les régions labélisées 1 et 2 dans la figure 7 e où la frontière est modélisée au minimum par cinq segments. Un critère naïf de fusion consiste alors à fusionner deux régions voisines dont la frontière commune ne respecte pas cet aspect rectiligne. Le nombre de segments $\delta_{\text {seg }}$ modélisant la frontière entre deux régions candidates à la fusion donné par le processus de caractérisation est donc exploité. Si le nombre de segments $\delta_{\text {seg }}$, qui a été déterminé de manière expérimentale, est supérieur à 1 $\left(\delta_{\text {seg }}>1\right.$ segment), les deux régions sont fusionnées. Cependant, ce critère de fusion peut favoriser une sous-segmentation de l'image à cause d'une mauvaise modélisation des frontières en segments suite à une non-détection de quelques objets d'intérêt délicats tels que certaines cheminées et fenêtres (ces frontières sont modélisées dans la plupart des cas par 3 ou 4 segments). A titre d'exemple, la non-détection de la fenêtre située entre les régions labélisées 3 et 4 de la figure $7 \mathrm{e}$ conduit à une caractérisation de la frontière commune en 4 segments. Pour pallier cet inconvénient, nous avons fait évoluer notre critère de fusion afin qu'il tienne compte d'informations supplémentaires sur les frontières et/ou sur les régions. L'adaptation du critère découle de l'observation suivante : la différence des moyennes intérieures $\bar{\varrho}_{R_{i}, R_{j}}$ de deux régions $R_{i}$ et $R_{j}$ situées de part et d'autre d'une frontière, séparant deux pans différents, est très inférieure au contraste $\xi_{R_{i}, R_{j}}$ observé à cette frontière (cf. les régions labélisées 2 et 3 dans la figure 7e). Par contre, cette différence $\bar{\varrho}_{R_{i}, R_{j}}$ est relativement proche du contraste $\xi_{R_{i}, R_{j}}$ aux frontières quand il s'agit de deux régions $R_{i}$ et $R_{j}$ appartenant au même pan d'une toiture. Par conséquent, l'information 
de contraste aux frontières et l'aspect rectiligne de ces dernières peuvent être utilisés pour définir un critère de fusion plus pertinent. Nous rappelons ici que les différents seuils ont été déterminés de manière expérimentale pour l'application visée mais méritent d'être recalculés si l'application venant à changer. Détaillons maintenant l'algorithme de fusion de régions :

- Im est l'image de gradient;

- $R_{i}$ et $R_{j}$ deux régions voisines candidates à la fusion;

- $p 1$ et $p 2$ deux pixels de l'image;

- $\delta_{\text {seg }}$ est le nombre de segments modélisant la frontière commune entre les régions $R_{i}$ et $R_{j}$;

- $\xi_{R_{i}, R_{j}}$ est le contraste à la frontière commune entre les régions $R_{i}$ et $R_{j}$;

$$
\xi_{R_{i}, R_{j}}=\frac{1}{\eta} \sum_{p 1 \in R 1} \max _{p 2 \in \mathcal{V}_{p 1}, p 2 \in R 2}(|\operatorname{Im}(p 1)-\operatorname{Im}(p 2)|)
$$

où $\eta$ est le nombre de pixels de la frontière commune entre les régions $R_{i}$ et $R_{j}$ et $\mathcal{V}_{p 1}$ est le voisinage de $\mathrm{p} 1$;

- $\bar{\varrho}_{R_{i}, R_{j}}$ est la différence des moyennes intérieures des régions $R_{i}$ et $R_{j}$.

$$
\bar{\varrho}_{R_{i}, R_{j}}=\left|\frac{1}{\eta_{1}} \sum_{p 1 \in R 1} \operatorname{Im}(p 1)-\frac{1}{\eta_{2}} \sum_{p 2 \in R 2} \operatorname{Im}(p 2)\right|
$$

où $\eta_{1}$ et $\eta_{2}$ représentent respectivement l'aire des régions $R_{i}$ et $R_{j}$.

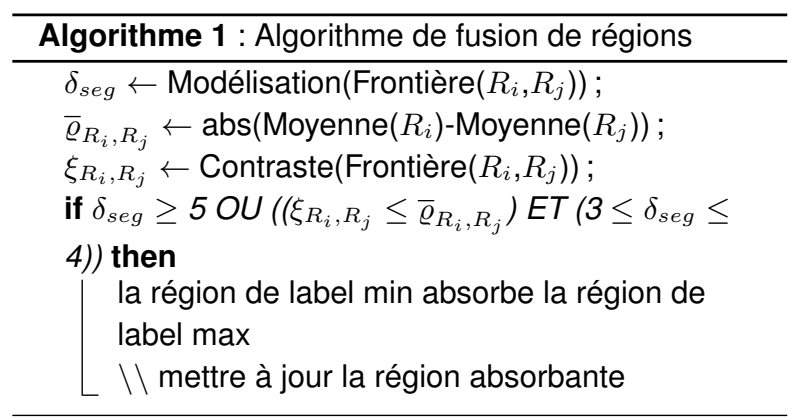

\subsection{Segmentation par LPE-contours}

Une fois la toiture segmentée en un nombre convenable de régions d'intérêt, nous cherchons à retrouver précisément les objets d'intérêt constituant celle-ci (cheminées, fenêtres, chiens assis, etc.). Pour ce faire, nous avons utilisé la LPE-contours introduite par VINCENT et SOILLE (Vincent et Soille, 1991), (Roerdink et Meijster, 2001) et qui fait partie des algorithmes de LPE par immersion. L'image de gradient utilisée en entrée de cet algorithme est identique à celle utilisée en entrée de la LPE-régions et permet de distinguer les zones homogènes et hétérogènes de l'image. Plus un pixel est situé dans une zone hétérogène, plus son gradient, et donc ici son élévation, est important. Lorsque

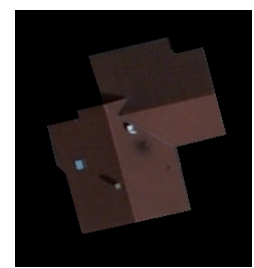

(a)

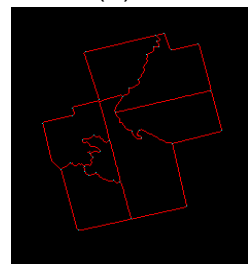

(d)

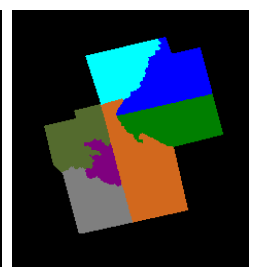

(b)

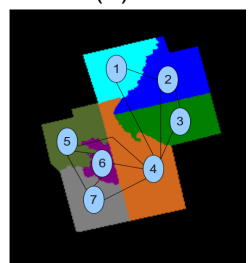

(e)

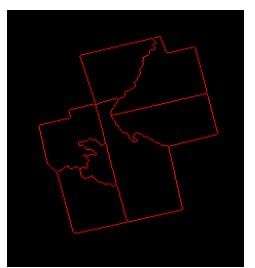

(c)

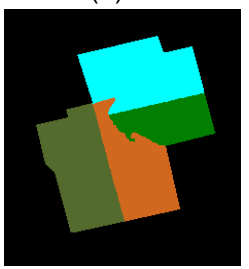

(f)
FIGURE 7: Illustration de la stratégie de fusion de régions. (a) image initiale, (b) image présegmentée par LPE, (c) extraction des frontières, (d) caractérisation par des segments, (e) RAG de l'image présegmentée, (f) segmentation finale.

deux bassins de rétention se rencontrent suite à une inondation à partir des minima du gradient, une Ligne de Partage des Eaux est créée pour les séparer. Le principe de cette LPE, que nous pouvons retrouvée dans (Roerdink et Meijster, 2001), est rappelé comme suit.

Soit $I: D \rightarrow \mathbb{N}$, une image en niveaux de gris, avec $h_{\min }$ et $h_{\max }$ les valeurs minimum et maximum de $I$. Les minima de $I$ sont associés à des bassins qui vont successivement grandir durant le procédé d'immersion. Soit l'ensemble $X_{h}$ l'union des bassins calculés au niveau $h$. Une composante connexe de la fonction seuil $Z_{h+1}$ de niveau $h+1$ peut être soit un minimum, soit une extension d'un bassin du niveau $X_{h}$. Le dernier cas entraîne le calcul de la zone d'influence géodésique de $X_{h}$ à l'intérieur de $Z_{h+1}$. Il en résulte la mise à jour de $X_{h+1}$. Soit l'ensemble $M I N_{h}$ l'union de tous les minima à l'altitude $h$. Le même bloc d'instruction pour un niveau $h$ variant de $h_{\min }$ à $h_{\max }$ :

$$
\left\{\begin{aligned}
X_{h_{\min }}= & \left\{p \in D / I(p)=h_{\min }\right\}=Z_{h_{\min }} \\
X_{h+1}= & M I N_{h+1} \cup\left(I Z_{Z_{h+1}}\left(X_{h}\right) \backslash Z_{h}\right), \\
& h \in\left[h_{\min }, h_{\max -1}\right]
\end{aligned}\right.
$$

où $p$ représente les coordonnées d'un pixel et le terme $\backslash Z_{h}$ de l'équation 11 assure qu'au niveau $h+1$, seuls les pixels dont l'intensité correspond à $h+1$ sont ajoutés aux bassins existants. $I Z_{A}(B)$ est l'union des zones d'influence géodésique des composantes connexes de $\mathrm{B}$ avec $\mathrm{B} \subseteq \mathrm{A}$. La ligne de partage des eaux de $I$ correspond au complément de $X_{h_{\max }}$ dans D :

$$
L P E(I)=D \backslash X_{h_{\max }}
$$

L'avantage d'effectuer une segmentation par LPEcontours est qu'elle conduit à des contours fins et fermés, mais génère cependant une sur-segmentation de l'image. La figure 8 illustre quelques résultats de segmentation obtenus avec l'algorithme de LPE-contours. II 
est important de noter que les différents objets d'intérêt sont quasiment tous détectés. La sur-segmentation produite ne constitue pas un obstacle pour la suite du traitement, car elle sera fortement réduite grâce au processus de coopération décrit dans la section suivante.

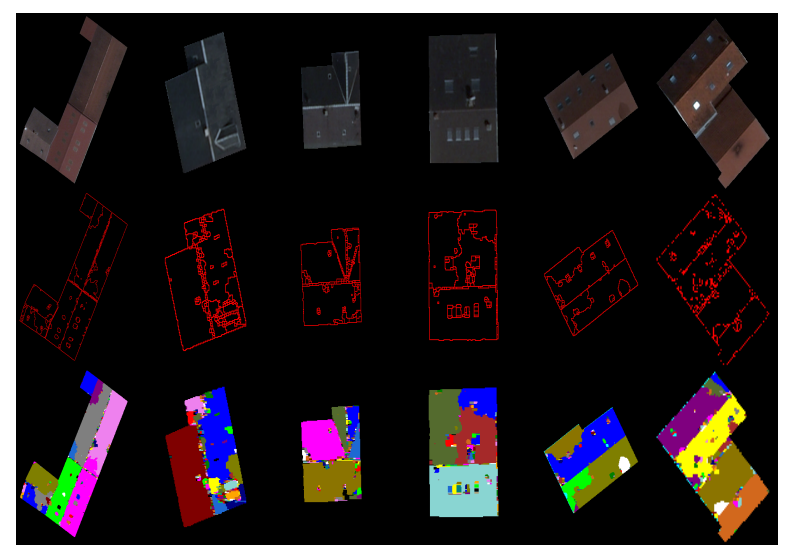

FIGURE 8: Exemples de segmentation obtenue par la LPEcontours (de haut en bas : l'image initiale, l'image segmentée par la LPE-contours et la carte de régions correspondante).

\section{Processus de coopération entre LPE-régions et LPE-contours}

Dans la section précédente, nous avons présenté deux méthodes de segmentation pour la détection de toitures à partir d'images aériennes : une LPE-régions couplée à une stratégie de fusion de régions afin de partitionner l'image en régions d'intérêt (les différents pans de toiture), et une LPE-contours permettant de détecter plus précisément les différents objets d'intérêt de la toiture (cheminées, fenêtres, chiens assis, etc.). Nous proposons, dans cette section, d'intégrer ces deux méthodes dans un schéma de segmentation coopératif dans l'objectif d'aboutir à un résultat de segmentation optimal. En effet, ce processus de coopération régions/contours exploite l'avantage de chacune des deux méthodes de segmentation mises en œuvre. Comme indiqué précédemment, la segmentation par LPE-régions couplée à notre stratégie de fusion de régions présente l'avantage de résoudre de manière pertinente le problème de sur-segmentation de l'image de toiture. En contre partie, elle s'accompagne d'une perte totale de certains objets d'intérêt tels que les cheminées, les fenêtres et certains pans de toiture. Cela s'explique par le fait que soit ces objets ne sont pas détectés lors de la phase de LPE-régions (à cause de l'absence de germes dans ces régions potentielles où d'un faible gradient ne permettant pas d'arrêter la croissance de régions), soit ils sont détectés mais perdus à la suite de l'étape de fusion de régions (car respectant le critère de fusion). La LPEcontours quant à elle permet de détecter la plupart des objets d'intérêt de la toiture, mais produit une forte sursegmentation sur les frontières entre les différents pans de toiture. Une coopération de ces deux techniques de segmentation semble alors intéressante afin d'améliorer la qualité de la segmentation globale. La figure 9 illustre les trois grandes étapes de ce processus de coopération que nous présentons ci-après :

1. La première étape, décrite dans la section 3 , consiste à prétraiter l'image initiale par un invariant colorimétrique en définissant un couple invariant/gradient adapté, l'image de gradient utilisée dans l'algorithme de LPE étant très sensible à l'invariant utilisé. Ce couple invariant/gradient optimal est défini et justifié dans la section 6 .

2. La deuxième étape consiste à effectuer en parallèle une segmentation de l'image prétraitée par une LPE-régions et par une LPE-contours. L'image produite par la LPE-régions est ensuite traitée par la technique de fusion de régions permettant de remédier au problème de sur-segmentation. L'image segmentée obtenue en sortie de ce traitement est alors étiquetée "image segmentée $A$ " dans le schéma de la figure 9. Parallèlement, l'image produite par la LPE-contours est soumise à une étape d'extraction des régions de sorte à ce que chaque contour fermé donne naissance à une région. A partir de l'image segmentée produite, étiquetée "image segmentée B" sur la figure 9, les barycentres des régions sont calculés. Une suppression des barycentres dont l'aire des régions est supérieure à un seuil est ensuite réalisée. Cette étape permet de ne garder que les barycentres des régions qui correspondent à la taille d'une fenêtre, d'une cheminée et de certains pans de toiture qui n'ont pas été détectés par la LPE-régions. Le seuil déterminé expérimentalement prend la valeur 600 (pixels).

3. La troisième et dernière étape traduit le processus de coopération entre la LPE-régions et la LPEcontours. Tout d'abord, une nouvelle LPE-régions est effectuée à partir des germes issus de la superposition des barycentres des images segmentées $A$ et $B$. Le résultat de cette segmentation, étiqueté "image segmentée C", met en évidence différents objets d'intérêt de la toiture qui n'ont pas été détectés par la LPE-régions avec fusion de régions (cf. l'étape 2). Ensuite, l'information sur les contours de l'image segmentée A est exploitée pour supprimer les petites régions présentes sur les frontières de l'image segmentée $\mathrm{C}$. Pour ce faire, nous supprimons toute région de l'image segmentée $C$ dont le barycentre coïncide avec un pixel contour de l'image segmentée A. Une LPE-régions est à nouveau effectuée à partir des germes correspondant aux barycentres des régions retenues afin de donner naissance à une nouvelle image segmentée étiquetée "image segmentée D". La segmentation finale est produite en superposant toutes les régions de l'image segmentée $A$ avec les régions de l'image segmentée $D$ dont l'aire est inférieure à un seuil défini de manière expérimentale et fixé ici à 800 (pixels). 


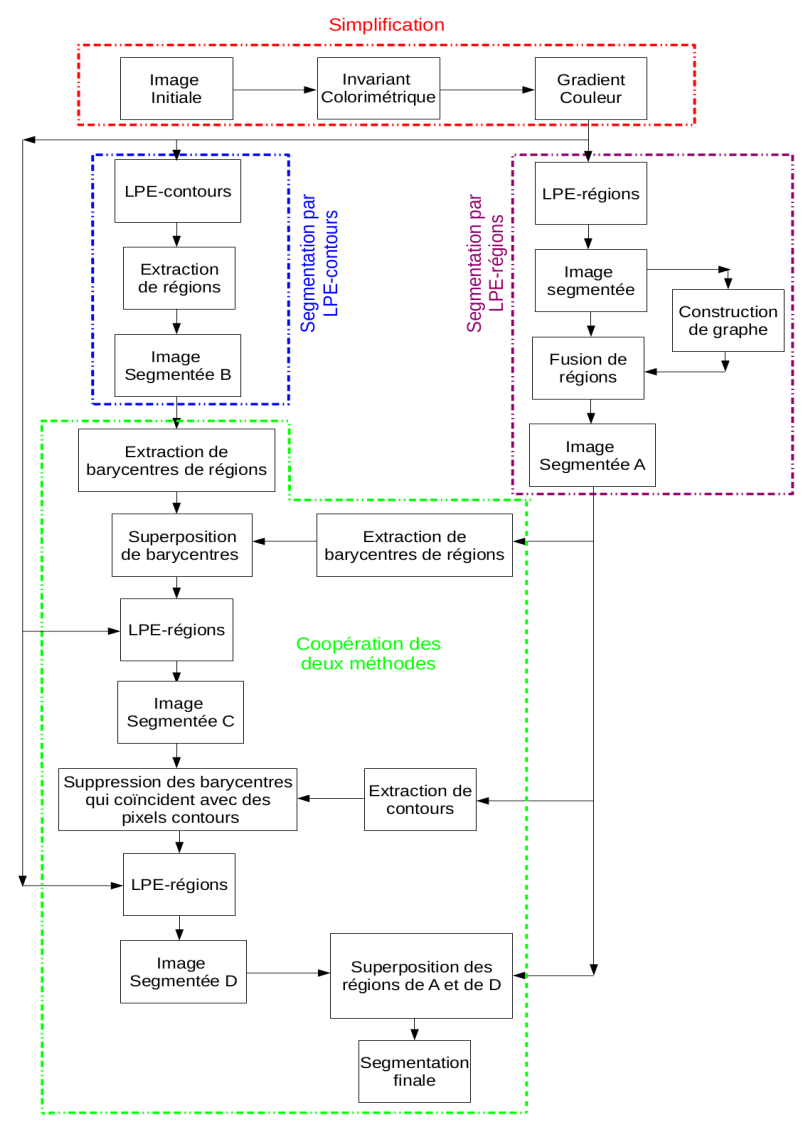

FIGURE 9: Synopsis du processus de coopération LPE-régions et LPE-contours.

La figure 10 illustre quelques résultats de segmentation à des étapes clés de l'approche de segmentation d'orthophotoplans proposée : (b) l'image segmentée obtenue par l'utilisation de la LPE-régions seule, (c) l'image étiquetée $A$ dans la figure 9 et obtenue après l'application de la stratégie de fusion de régions, (d) l'image étiquetée B obtenue avec la LPE-contours, (e) l'image étiquetée $C$ obtenue par une LPE-régions effectuée à partir des germes issus de la superposition des barycentres des images étiquetées $A$ et $B$, (f) l'image étiquetée $D$ obtenue après l'étape de suppression de petites régions, et enfin $(\mathrm{g})$ la segmentation finale obtenue après superposition des régions d'intérêt (cheminée, fenêtre, chiens assis, etc) de l'image étiquetée $D$ avec toutes les régions de l'image étiquetées $A$.

\section{Résultats expérimentaux}

Dans cette section, nous présentons les résultats expérimentaux des différentes étapes de l'approche proposée, à savoir :

1. Le choix du couple optimal invariant/gradient dans l'étape de prétraitement;

2. La performance de la stratégie de fusion associée à la LPE-régions ainsi que celle de l'algorithme de LPE-contours ;

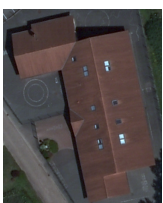

(a)

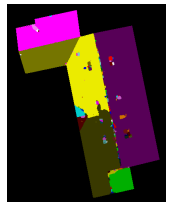

(e)

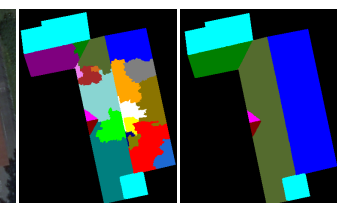

(b)

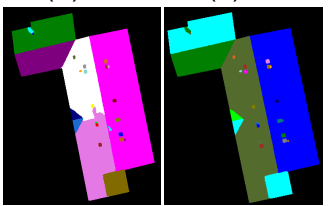

(f) (g)

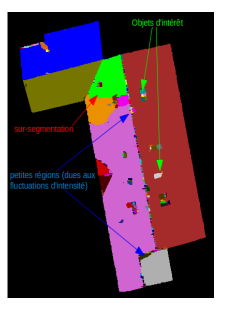

(d)
FIGURE 10: Etapes clés du processus de coopération. (a) toiture, (b) image segmentée par LPE-régions seule, (c) image obtenue après fusion de régions (l'image étiquetée $A$ sur la figure 9), (d) image segmentée par LPE-contours ('limage étiquetée B), (e) l'image étiquetée $\mathrm{C}$, (f) l'image étiquetée $\mathrm{D}$ et (g) segmentation finale.

3. Les résultats de segmentation suite au processus de coopération LPE-régions et LPE-contours.

Les tests présentés dans ce papier ont été effectués sur un orthophotoplan de résolution de 10000 pixels $\times 10000$ pixels (avec un pixel $=16 \mathrm{~cm}$ ) fourni par la Communauté de l'Agglomération Belfortaine (CAB 2008) constitués de différentes zones d'habitation (quartiers résidentiels, campagne, infrastructures routières, etc) et/ou industrielles avec différents type de matériaux composants la toiture. Les 100 toitures extraites de cet orthophotoplan à partir de l'empreinte au sol fournie ont été segmentées puis évaluées avec le critère de VINET (Vinet, 1991) par rapport à une segmentation de référence. Pour cela, un expert (ayant connaissance de l'objectif, à savoir segmenter précisément les différents éléments constituant la toiture) a réalisé une segmentation fine de chaque toiture à la main. Chaque région identifiée/créée a reçue un label/une couleur différente pour la dissocier de ses voisines.

\subsection{Choix d'un couple optimal invariant/gradient}

Comme indiqué dans la section 3, nous proposons de prétraiter l'image initiale par un invariant colorimétrique. Cependant, cet invariant étant directement appliqué sur l'image initiale, le résultat du calcul du gradient utilisé en entrée de la LPE est influencé par l'invariant utilisé. C'est pour cette raison que nous cherchons à définir le meilleur couple invariant/gradient. Nous utilisons donc l'information disponible en niveaux de gris (composante couleur invariant/gradient niveaux de gris) mais aussi en couleur (invariant/gradient couleur). Les figures 11 et 12 illustrent respectivement les meilleurs couples composante couleur invariant colorimétrique/gradient niveaux de gris et les meilleurs couples invariant colorimétrique/gradient couleur. Pour plus de lisibilité, nous nous sommes contentés de ne présenter sur ces graphiques que les résultats obtenus sur 18 invariants représentatifs parmi les 24 invariants colorimétriques énoncés dans la section 3 . 
En considérant l'information de niveaux de gris, nous montrons que les résultats de segmentation dépendent grandement de la composante couleur sélectionnée (cf. figure 11). En effet, pour les trois composantes $X, Y$ et $Z$ des invariants Greyworld, normalisation affine (notée Affine dans les figures 11,12), Maximum-Intensité (noté Mintensity dans les figures 11, 12), MaxRGB et Rgbrang, les valeurs de VINET sont élevées (ce qui implique que les résultats sont meilleurs) par rapport aux composantes couleur des autres invariants colorimétriques, et ce, pour la plupart des gradients niveaux de gris utilisés à l'exception de Shen et de Deriche. Ceci montre clairement que pour notre application, ces invariants sont les plus significatifs. II est aussi important de noter que seulement la composante $X$ de la normalisation L2 (L2-X) et la composante $Z$ de l'espace hsl (hsl-Z) donnent les meilleurs résultats de segmentation. Le tableau 2 illustre, pour une meilleure compréhension, les valeurs moyennes de Vinet obtenues en fonction de quelques couples composante couleur d'un invariant colorimétrique/gradient niveaux de gris. Nous pouvons alors conclure en définissant un ordre de préférence sur l'utilisation d'un couple composante invariant colorimétrique/gradient niveaux de gris : HSLZ/Prewitt, HSL-Z/Sobel, HSL-Z/GradientM, MintensityY/Sobel, L2-X/Sobel.

\begin{tabular}{|c|c|c|c|c|}
\hline Gradient & GradientM & Sobel & Prewitt & Gradient \\
\hline$R G B-X$ & $67,6 \%$ & $69,8 \%$ & $69,2 \%$ & $73,3 \%$ \\
\hline$R G B-Y$ & $69,9 \%$ & $80,8 \%$ & $78 \%$ & $79 \%$ \\
\hline$R G B-Z$ & $69,3 \%$ & $70,7 \%$ & $70,2 \%$ & $71 \%$ \\
\hline$L 2-X$ & $72,3 \%$ & $82,5 \%$ & $82 \%$ & $81,8 \%$ \\
\hline Mintensity- $Y$ & $70,8 \%$ & $82,8 \%$ & $81,9 \%$ & $79,8 \%$ \\
\hline$R G B-$ rang & $61 \%$ & $77 \%$ & $59,5 \%$ & $80,6 \%$ \\
\hline$H S L-Z$ & $76,7 \%$ & $\mathbf{8 8} \%$ & $\mathbf{8 8 , 4} \%$ & $\mathbf{8 7 . 9} \%$ \\
\hline$H S L-X$ & $48,2 \%$ & $49,2 \%$ & $49 \%$ & $48,9 \%$ \\
\hline$H S L-Y$ & $57 \%$ & $54,8 \%$ & $55,5 \%$ & $55,6 \%$ \\
\hline
\end{tabular}

TABLE 2: Taux de segmentation calculés avec le critère de Vinet en fonction des couples composante couleur (d'invariant colorimétrique)/gradient niveaux de gris.

En considérant l'information couleur, nous montrons que l'utilisation des invariants colorimétriques Greyworld, normalisation affine, Maximum-Intensité, MaxRGB et Rgb-rang donnent de bons résultats de segmentation, et ce quel que soit le gradient couleur utilisé (cf. figure 12). Le tableau 3 illustre, pour une meilleure compréhension, les valeurs moyennes de Vinet obtenues en fonction de quelques couples invariant colorimétrique /gradient couleur. Comme nous l'avons fait en niveaux de gris, nous pouvons alors définir un ordre de préférence sur l'utilisation d'un couple invariant colorimétrique/gradient couleur : Greyworld/Di-Zenzo, Mintensité/Di-Zenzo, MaxRGB/Di-Zenzo, normalisation affine/Di-Zenzo, Mintensité/GradientC. Dans le cadre de notre application de détection de toitures à partir d'images aériennes, nous avons retenu, l'utilisation du couple Greyworld/Di-zenzo pour le prétraitement de l'image et le calcul du gradient intervenant dans l'algorithme de
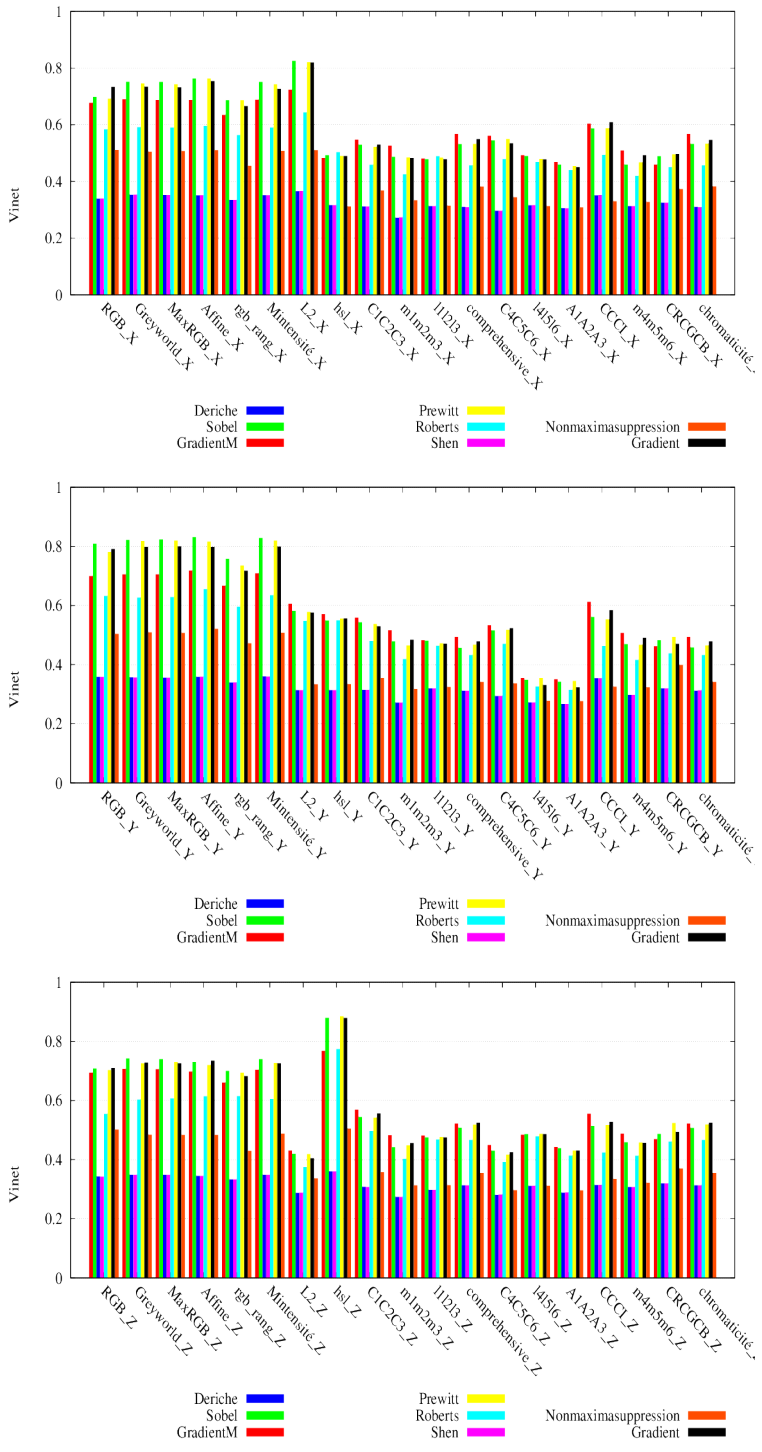

FIGURE 11: Résultats de segmentation de l'image en niveau de gris effectuée avec différents couples composante couleur invariant colorimétrique/gradient niveau de gris (de haut en bas : les résultats de segmentation sur la composante $X$ (haut), la composante $\mathrm{Y}$ (milieu), la composante $Z$ (bas)).

LPE.

\begin{tabular}{|c|c|c|c|c|}
\hline Gnvariant & Carron & Dizenzo & MGradient & GradientC \\
\hline RGB & $71,3 \%$ & $\mathbf{8 3 . 5} \%$ & $61,6 \%$ & $\mathbf{8 3 , 8} \%$ \\
\hline Greyworld & $75,2 \%$ & $\mathbf{8 5} \%$ & $63 \%$ & $\mathbf{8 4 , 7 \%}$ \\
\hline MaxRGB & $75,5 \%$ & $\mathbf{8 4 , 5} \%$ & $63,3 \%$ & $\mathbf{8 4 , 6 \%}$ \\
\hline$M$-intensity & $76 \%$ & $\mathbf{8 4} \%$ & $63,3 \%$ & $\mathbf{8 4 , 5} \%$ \\
\hline$R G B$-rang & $61 \%$ & $77 \%$ & $59,5 \%$ & $80,6 \%$ \\
\hline$N$-affine & $75 \%$ & $\mathbf{8 4 , 5} \%$ & $63,8 \%$ & $\mathbf{8 4 , 1} \%$ \\
\hline$c_{1} c_{2} c_{3}$ & $55 \%$ & $60 \%$ & $51,6 \%$ & $52,4 \%$ \\
\hline$m_{1} m_{2} m_{3}$ & $46 \%$ & $54 \%$ & $49 \%$ & $52 \%$ \\
\hline$l_{1} l_{2} l_{3}$ & $52 \%$ & $47 \%$ & $44,7 \%$ & $25,5 \%$ \\
\hline
\end{tabular}

TABLE 3: Taux de segmentation calculés avec le critère Vinet en fonction des couples invariant colorimétrique/gradient couleur. 


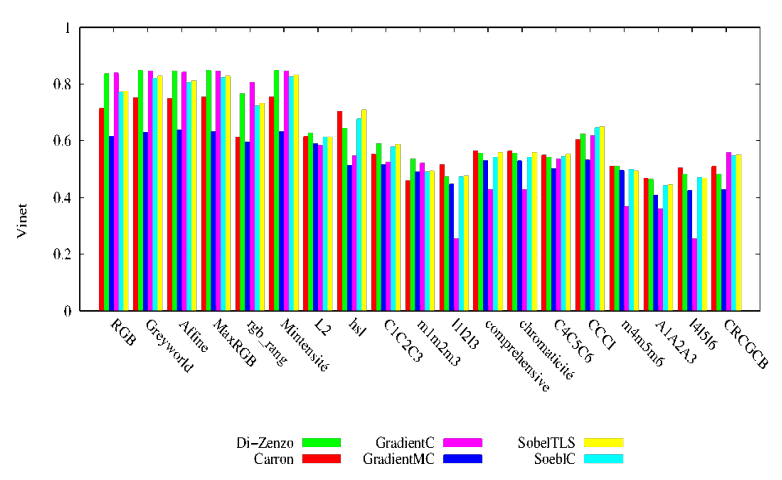

FIGURE 12: Résultats de segmentation de l'image couleur effectuée avec différents couples invariant colorimétrique/gradient couleur.

\subsection{Performance de l'algorithme de fusion de ré- gions}

Afin de montrer la robustesse et la fiabilité de la procédure de fusion de régions proposée pour résoudre le problème de sur-segmentation engendrée par la LPErégions, nous présentons sur la figure 13 , les résultats de segmentation sans et avec fusion de régions. Nous pouvons constater alors que la stratégie de fusion de régions permet d'améliorer les résultats de segmentation par rapport à ceux obtenus avec une LPErégions seule. En effet, la valeur de VINET est plus élevée lorsque l'on associe la stratégie de fusion de régions à l'étape de segmentation par LPE-régions. L'application de cette stratégie fait passer le taux de bonne segmentation de $83 \%$ (LPE-régions seule) à 94\% (LPE-régions + fusion). Ceci peut être expliqué par le fait que la stratégie de fusion a permis de fusionner la plupart des régions non-significatives issues de la sur-segmentation, tout en préservant les pans de toitures.

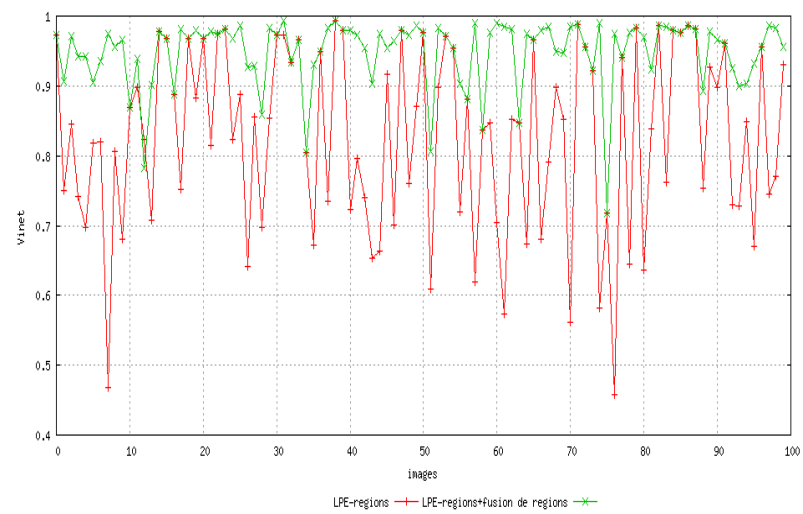

FIGURE 13: Résultats de segmentation obtenus avec la LPErégions seule (en rouge) et avec la stratégie de fusion de régions (en vert).

La figure 14 illustre quelques résultats de segmentation et met en évidence le gain apporté par la stratégie de fusion de régions. A titre d'exemple, si l'on considère la première image (première colonne de la figure 14), le taux de bonne segmentation obtenu avec la fusion de régions est de $97 \%$ VS $47 \%$ avec la LPE-régions seule.
Visuellement, nous pouvons constater que l'utilisation de la LPE-régions seule implique une importante sursegmentation de l'image, corrigée de manière pertinente par l'application de l'étape de fusion de régions. Cependant, cette correction s'accompagne d'une perte de certains objets d'intérêt tels que les cheminées, fenêtres, etc. Nous montrons dans la section suivante que cette perte peut être compensée par le processus de coopération des méthodes de segmentation LPE-régions et LPEcontours.

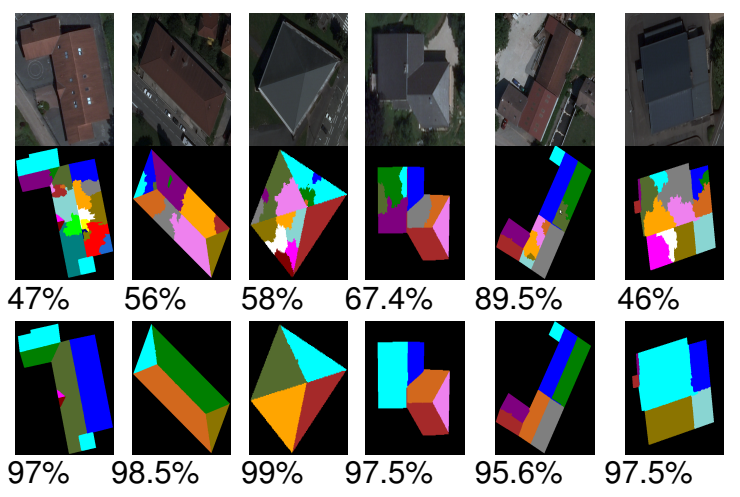

FIGURE 14: Résultats de segmentation obtenus avec la stratégie de fusion de régions proposée (de haut en bas, les images initiales, les images obtenues par la LPE-régions seule et les segmentations obtenues après l'application de la stratégie de fusion de régions). Les valeurs en dessous des images représentent les taux de bonne segmentation.

\subsection{Coopération LPE-régions/LPE-contours}

Dans cette section, nous présentons les résultats de segmentation de l'approche globale proposée. Afin de mettre en évidence l'importance des différentes étapes de notre approche (synopsis présenté sur la figure 9), nous présentons sur les figures 15 et 16, les résultats de segmentation obtenus par LPE-régions seule (en rouge sur la figure 15), par LPE-contours (en vert sur la figure 15) et par le processus de coopération (en bleu sur la figure 15). Nous pouvons constater que l'approche de coopération proposée permet d'aboutir aux meilleurs résultats de segmentation.

En effet, en moyenne, la différence est importante puisque nous passons de $83 \%$ pour la LPE-régions, ou $88 \%$ pour la LPE-contours à $96 \%$ pour la coopération LPE-régions/LPE-contours. Nous présentons ces résultats sur la figure 16 en illustrant sur quelques images, les étapes clés du processus de coopération proposé. Notons que les résultats de segmentation obtenus par la LPE-régions seule ne sont pas satisfaisants puisque les images sont sur-segmentées (cf. $2^{\text {ème }}$ ligne de la figure 16). La procédure de fusion de régions permet d'améliorer ces résultats, avec la perte de certains objets d'intérêt de la toiture comme les fenêtres et les cheminées (cf. $3^{\text {ème }}$ ligne de la figure 16). La LPE-contours seule permet de conserver ces objets mais produit une sur/sous-segmentation importante (cf. $4^{\text {ème }}$ ligne de la figure 16). La procédure de coopération proposée 
exploite ainsi les avantages de chacune des méthodes et permet alors d'obtenir les meilleurs résultats. En effet, les pans de toitures sont bien segmentés et la plupart des fenêtres et cheminées sont présentes (cf. $5^{\text {ème }}$ ligne de la figure 16).

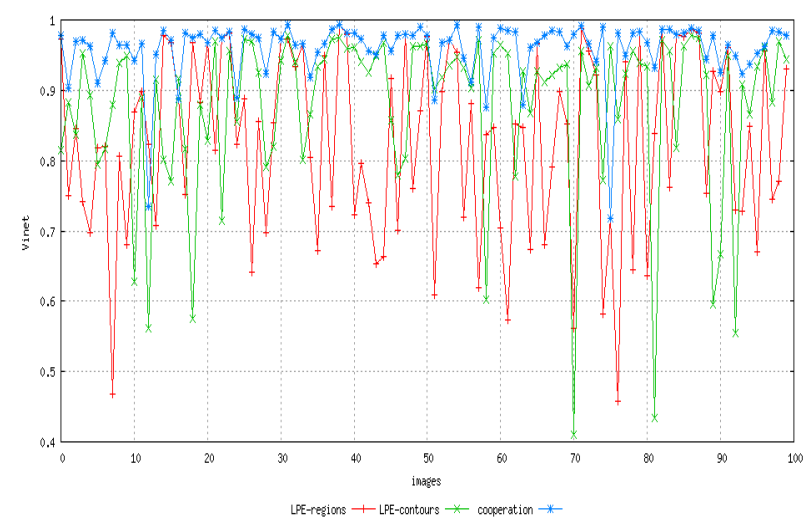

FIGURE 15: Résultats de l'évaluation des images segmentées par la LPE-régions (en rouge), par la LPE-contours (en vert) et par le processus de coopération (en bleu).

Il convient toutefois de noter, que pour certaines images (5 sur les 100 images testées), notre approche fournit des résultats moins performants que ceux obtenus par la LPE-régions et la LPE-contours appliquées séparément. En effet, nous pouvons constater sur la figure 13 que les résultats de notre approche sont en dessous de ceux de la LPE-contours pour 4 images et de ceux de la LPE-régions pour une image. La figure 17 illustre un exemple pour lequel la qualité de la segmentation obtenue par la procédure de coopération est moins bonne en comparaison avec l'utilisation de la LPE-contours seule. Nous montrons ainsi que la procédure de coopération souffre de la non-détection/perte d'un pans de toiture, alors que la LPE-contours a pu le détecter. Cela s'explique comme indiqué précédemment dans la section 5, par le fait que ce pans de toiture n'est pas détecté par la LPE-régions suite à l'absence du germe correspondant. II est donc naturel qu'il ne figure pas sur l'image segmentée générée par l'approche proposée puisque celle-ci prend en compte le résultat de segmentation produit par la LPE-régions. Pour les autres images, il s'agit de toitures très complexes, composées de pans avec des blocs juxtaposés ou accolés (et difficilement identifiables pour l'être humain). La méthode de fusion de régions fusionne donc à tord les régions de ces pans difficiles puisque la frontière est de mauvaise qualité. Par conséquent, certains pans de toitures ne seront pas présent dans le résultat final de segmentation par coopération alors qu'ils étaient détectés avec les algorithmes de segmentation LPE-régions et LPE-contours seuls.

\section{Conclusion}

Dans cet article, nous avons proposé une méthode de segmentation par coopération LPE-régions et

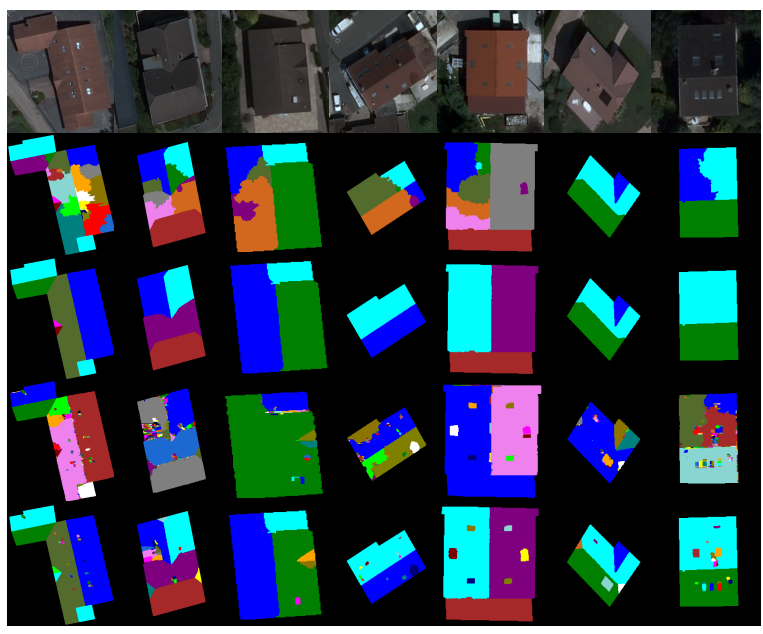

FIGURE 16: Résultats de segmentation basés sur la procédure de coopération proposée. De haut en bas, les images initiales, les images obtenues par la LPE-régions, après l'étape de fusion de régions, par la LPE-contours et par la procédure de coopération.
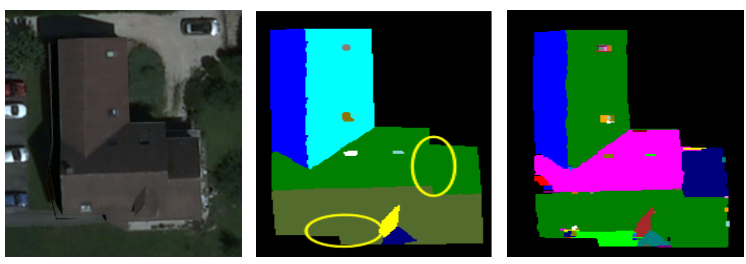

FIGURE 17: llustration d'une image pour laquelle la segmentation produite par notre approche (l'image de la deuxième colonne) moins satisfaisante que celle réalisée avec la LPEcontours (l'image de la troisième colonne).

LPE-contours pour la détection de toitures à partir d'images aériennes (orthophotoplans). Cette méthode intègre deux types de segmentation (régions et contours) afin de tirer partie des avantages de chaque type de segmentation et permettre une meilleure prise en compte des caractéristiques des objets d'intérêt présents dans l'orthophotoplan. Avant de procéder aux différentes segmentations, une première étape consiste à prétraiter l'image initiale par l'application d'un invariant colorimétrique adapté. Ce prétraitement de l'image initiale est effectué en déterminant un gradient optimal associé, nécessaire aux LPE-régions et LPE-contours. Une stratégie de fusion de régions est aussi proposée afin de réduire le problème de sur-segmentation liée à la LPE-régions. Les résultats de segmentation obtenus par l'approche de coopération proposée sont très satisfaisants puisque nous obtenons un taux de bonne segmentation de $96 \%$ avec la procédure de coopération contre $83 \%$ avec l'utilisation d'une LPE-régions seule ou $88 \%$ avec une LPEcontours seule.

Bien que l'approche proposée enregistre des très bonnes performances face à la problématique étudiée, il convient de mentionner une limite majeure de l'approche proposée. En effet, à l'instar de la plupart des méthodes de segmentation de la littérature, la méthode de segmentation d'orthophotoplan proposée dans ce papier reste 
dépendante de la résolution des images traitées. En effet, la méthode reste robuste et pertinente face à des images de même résolution, mais en contre partie, elle devient une méthode paramétrique qui nécessite de définir le meilleur couple invariant/gradient dans l'étape de prétraitement de l'image, d'ajuster les paramètres alpha et beta de la technique de réduction de germes ainsi que la taille des petites structures caractérisant la toiture optimisées à partir de plusieurs tests et expérimentations. Comme perspective à ces travaux, nous travaillons actuellement sur l'extraction automatique des imagettes de toitures à partir de l'orthophotoplan fourni et constitué de route, jardins, habitations, etc. sans avoir besoin de connaître l'empreinte au sol des bâtiments.

\section{Références}

Angiati, E., Dellepiane, S., 2011. Identification of roofs perimeter from aerial and satellite images. 17th International Conference on Digital Signal Processing. Island of Corfu, Greece, $1-7$.

Anil, P., Natarajan, D., 2010. A novel approach using active contour model for semi-automatic road extraction from high resolution satellite imagery. International Conference on Machine Learning and Computing. Bangalore, India, 263-266.

Bellet, F., Salotti, M., Garbay, C., 1995. Une approche opportuniste et coopérative pour la vision de bas niveau. Traitement du signal $12, n^{\circ} 5,479-494$.

Buchsbaum, G., 1980. A spatial processor model for object colour perception. Journal of the Franklin Institute $310, n^{\circ} 1$, $1-26$.

Carron, T., 1995. Segmentation d'images couleur dans la base teinte luminance saturation : approche numérique et symbolique. Thèse de doctorat de l'Université de Savoie.

Chu, C., Aggarwal, J., 1993. The integration of image segmentation maps. using region and edge information. IEEE Transactions on Pattern Analysis and Machine Intelligence 15, $\mathrm{n}^{\circ}$ 12, 1241-1252.

Cohen, A., Attia, D., Meurie, C., Ruichek, Y., 2010. Une méthode de segmentation hybride par combinaison adaptative des informations texture et couleur. MAJESTIC, Bordeaux France.

Cong, T., Khoudour, L., Achard, C., Meurie, C., Lezoray, O., 2010. People re-identification by spectral classification of silhouettes. Signal Processing 90, n8, 2362-2374.

Dargham, J., 2008. Lip detection by the use of neural networks. Artificial Life and Robotics 12, $n^{\circ} 1-2,301-306$.

Di-Zenzo, R., 1986. A note on the gradient of a multi-image. Computer Vision, Graphics and Image Processing 33, $n^{\circ} 1$, 116-125.

El-merabet, Y., Meurie, C., Ruichek, Y., Sbihi, A., Touahni, R., 2011a. Orthophotoplan segmentation and colorimetric invariants for roof detection. 16th International Conference on Image Analysis and Processing, LNCS 6979. Ravenna, Italy, 394-403.

El-merabet, Y., Meurie, C., Ruichek, Y., Sbihi, A., Touahni, R., 2011b. Watershed regions and watershed lines based cooperation strategy for image segmentation. application to roof detection. IEEE International Symposium on Signal Processing and Information Technology. Bilbao, Spain, 393-398.

El-merabet, Y., Meurie, C., Ruichek, Y., Sbihi, A., Touahni, R., 2012. Orthophotoplan segmentation based on regions merging for roof detection. IS\&T/SPIE Electronic Imaging-Image Processing : Machine Vision Applications V. Burlingame, California, USA, 830000.

Finlayson, G., Hordley, D., Schaefer, G., Tian, G., 2005. Illumi- nant and device invariant colour using histogram equalization. Pattern Recognition 2, $n^{\circ}$ 38, 179-190.

Fusiello, A., Trucco, E., Tommasini, T., Roberto, V., 1999. Improving feature tracking with robust statistics. Pattern Analysis \& Applications $2, n^{\circ} 4,312-320$.

Gevers, T., Smeulders, A., 1997. Object recognition based on photometric colour invariants. 10th Scandinavian conference on image analysis. Lappeenranta, Finland, 861-868.

Gevers, T., Smeulders, A., 1999. Colour based object recognition. Pattern Recognition 3, n³2, 453-464.

Gevers, T., Stockman, H., 2004. Robust histogram construction from color invariants for object recognition. IEEE Transactions on Pattern Analysis and Machine Intelligence 1, $n^{\circ} 26,113$ 118.

Golland, P., Bruckstein, A., 1997. Motion from color. Computer Vision and Image Understading 68, $n^{\circ} 3,346-362$.

Gouiffès, M., 2005. Apports de la couleur et des modèles de réflexion pour l'extraction et le suivi de primitives. Thèse de doctorat de l'Université de Poitiers.

Grimaud, M., 1992. A new measure of contrast : the dynamics. SPIE'92 Image Algebra and. Morphological Image Processing III. San Diego, USA 1769, 292-305.

Hazelhoff, L., With, P. N., 2011. Localizations of buildings with a gable roof in very-high-resolution aerial images. SPIE Electronic Imaging, San Francisco, USA 7882.

Jayadevappa, D., Kumar, S., Murthy, D., 2009. A hybrid segmentation model based on watershed and gradient vector flow for the detection of brain tumor. International journal of Signal processing, Image processing and Pattern Recognition 2, $n^{\circ} 3,29-42$.

Kermad, C., Chehdi, K., 1998. Segmentation d'images : mise en oeuvre automatique par coopération de méthodes. Traitement du Signal 15, $n^{\circ} 4,321-336$.

Latecki, L., Rajagopal, V., Gross, A., 2005. Image retrieval and reversible illumination normalization. IS\&T/SPIE. Internet Imaging VI. San Jose, USA.

Lezoray, O., Elmoataz, A., Cardot, H., Revenu, M., 2007. Segmentation d'images couleur : applications en microscopie cellulaire. Traitement du Signal 17, $\mathrm{n}^{\circ}$ 1, 33-45.

Liu, Y., Yu, Y., 2012. Interactive image segmentation based on level sets of probabilities. IEEE Transactions on Visualization and Computer Graphics 18, n ${ }^{\circ} 2,202-213$.

Meyer, F., 1991. Un algorithme optimal de ligne de partage des eaux. 8ème Congrès RFIA, Lyon-Villeurbanne, France 2, 847-857.

Meyer, F., Beucher, S., 1990. Morphological segmentation. Visual Communication and Image Representation 1, $n^{\circ} 1,21-$ 46.

Mueller, M., Segl, K., Kaufmann, H., 2004. Edge- and regionbased segmentation technique for the extraction of large, man-made objects in high-resolution satellite imagery. Pattern Recognition 37, $n^{\circ} 8,1619-1628$.

Muñoz, X., Cufí, X., Freixenet, J., Martí, J., 2000. new approach to segmentation based on fusing circumscribed contours, region growing and clustering. IEEE International Conference on Image Processing. Vancouver, BC, Canada 1, 800-803.

Roerdink, J. B. T. M., Meijster, A., 2001. The watershed transform : Definitions, algorithms and parallelization strategies. Fundamenta Informaticae 41, $\mathrm{n}^{\circ} 1-2,187-228$.

Salotti, J., 1994. Gestion des informations dans les premières étapes de la vision par ordinateur. Thèse de doctorat, Institut national polytechnique de Grenoble.

Schaefer, G., 2004. How useful are colour invariants for image retrieval. Second International Conference, Computer Vision and Graphics, Warsaw, Poland, 381-386.

Sebari, I., He, D., 2007. Les approches de segmentation d'image par coopération régions-contours. Revue Télédétection $7, n^{\circ} 1-2-3-4,499-506$. 
Sebari, I., He, D., 2009. Approach to nonparametric cooperative multiband segmentation with adaptive threshold. Applied Optics 48, n²0, 3967-3978.

Sirmacek, B., Unsalan, C., 2008. Building detection from aerial imagery using invariant color features and shadow information. 23rd International Symposium on Computer and Information Sciences, Istanbul, Turkey, 1-5.

Smet, P. D., Pires, R., Vleeschauwer, D., Bruyland, I., 2000. Implementation and analysis of an optimized rainfalling watershed algorithm. IS\&T/SPIE's 12th Annual Symposium Electronic Imaging. San Jose, California USA 3971, 759-766.

Vincent, L., Soille, P., 1991. Watersheds in digital spaces. an efficient algorithm based on immersion simulations. IEEE Trans. Pattern Analysis and Machine Intelligence 13, $n^{\circ} 6,583-598$.

Vinet, L., 1991. Segmentation et mise en correspondance de régions de paires d'images stérioscopiques. Thèse de doctorat, de l'Université de Paris IX Dauphine.

Volodymyr, M., Geoffrey, E., 2010. Learning to detect roads in high-resolution aerial images. 11th European conference on Computer vision. Crete, Greece, 210-223.

Yan, J., Zhao, B., Wang, L., Zelenetz, A., Schwartz, L., 2006. Marker-controlled watershed for lymphoma segmentation in sequential ct images. Medical Physics 33, $n^{\circ} 7,2452-2460$.

Zugaj, D., Lattuati, V., 1998. A new approach of color images segmentation based on fusing region and edge segmentations outputs. Pattern Recognition 31, $n^{\circ} 2,105-113$. 